Revue d'histoire de l'Amérique française

ZRE REVUE D.HISTOIRE DE L'AMÉRIQUE FRANÇAISE

\title{
La tenure seigneuriale a-t-elle été abolie par suite des plaintes des censitaires?
}

\section{Georges Baillargeon}

Volume 21, numéro 1, juin 1967

URI : https://id.erudit.org/iderudit/302645ar

DOI : https://doi.org/10.7202/302645ar

Aller au sommaire du numéro

Éditeur(s)

Institut d'histoire de l'Amérique française

ISSN

0035-2357 (imprimé)

1492-1383 (numérique)

Découvrir la revue

Citer cet article

Baillargeon, G. (1967). La tenure seigneuriale a-t-elle été abolie par suite des plaintes des censitaires ? Revue d'histoire de l'Amérique française, 21(1), 64-80. https://doi.org/10.7202/302645ar d'utilisation que vous pouvez consulter en ligne.

https://apropos.erudit.org/fr/usagers/politique-dutilisation/ 


\section{LA TENURE SEIGNEURIALE A-T-ELLE ÉTÉ ABOLIE PAR SUITE DES PLAINTES DES CENSITAIRES ? *}

Pour répondre à cette question, nous allons voir, étape par étape, comment s'est opérée cette abolition, car les erreurs commises par ceux qui se sont aventurés à parler de ce sujet - en pensant qu'il était très simple - viennent de deux causes principales: $1^{\circ}$ du manque de connaissance des circonstances qui ont précédé, accompagné et suivi non pas l'abolition, mais les diverses mesures d'abolition; $2^{\circ}$ des extrapolations injustifiées et injustifiables qui ont fait appliquer à toutes les seigneuries des témoignages qui convenaient à une, à quelques-unes ou même à plusieurs d'entre elles.

Pour définir nos termes disons tout d'abord que ce que nous entendons ici par l'abolition du régime seigneurial, ce n'est pas seulement 1854. Il y a eu plusieurs lois visant à abolir le régime seigneurial et chacune d'elles devait, dans la pensée du législateur, parvenir, à plus ou moins longue échéance, à l'abolition de ce régime. Chacune d'elles a appris aux dirigeants de l'époque un certain nombre de vérités, leur a révélé un certain nombre de problèmes insoupçonnés. Ainsi, on croyait qu'il suffirait de permettre aux gens de racheter les droits seigneuriaux pour se débarrasser de ce régime. Les faits ont prouvé le contraire. Mais il fallait essayer pour le savoir. Peu à peu une réalité s'est imposée à tous: l'abolition du régime seigneurial était une chose infiniment plus facile à dire qu'à faire. La question du jour était: comment procéder ? Même des gens très intelligents, comme il y en avait au Canadien par exemple,

* Travail présenté à la réunion générale de l'Institut d'Histoire de l'Amérique française, le 15 avril 1967. 
ne voyaient absolument aucune solution acceptable aux deux parties. Et quand quelqu'un parlait de l'abolition, on lui disait: Très bien, mais quel plan avez-vous à nous suggérer ?

Les lois visant à l'abolition se divisent en deux catégories: la première comprend les lois adoptées par le parlement impérial, la seconde comprend les lois adoptées par la législature de la Province.

La première étape dans l'abolition de la tenure seigneuriale est la loi impériale de 1822, appelée Acte du commerce du Canada. ${ }^{1}$ La permission de racheter ou, comme on disait alors, de commuer les lods et ventes dans les seigneuries de la Couronne, en particulier dans les villes de Québec et de Trois-Rivières, est accordée cette année-là presque par accident. Le seigneur Edward Ellice, ayant demandé l'adoption d'une loi qui lui permît d'obtenir la propriété absolue de ses terres, ${ }^{2}$ le secrétaire chargé de la rédaction du projet de loi consulte à ce sujet un Canadien d'origine britannique, le juge en chef James Monk, de passage à Londres. Celui-ci, qui est un résident de Québec, en profite pour travailler dans son propre intérêt. Il conseille d'ajouter dans le projet de loi une clause permettant aux censitaires de la Couronne de se libérer. ${ }^{3}$ C'est ainsi que les gens de Québec et de Trois-Rivières en particulier, nommément celui qui avait eu la présence d'esprit de suggérer l'addition de cette clause, peuvent, dès 1822 , racheter les droits seigneuriaux, ${ }^{4}$ alors qu'Ellice, qui a soulevé la question, n'obtient pour luimême - et les autres seigneurs - qu'une clause incomplète, applicable seulement en certains cas. ${ }^{5}$ 121.

1 Acte du Commerce du Canada, 5 août 1822, DRHC, 1819-1828, 120-

2 Témoignage d'Edward Ellice, 15 mai 1828, Appendice du XXXVIIIe volume des Journaux de la Chambre d'Assemblée du Bas-Canada, 2 , Appendice HH, [n.p.]; R. Wilmot à Dalhousie, 12 août 1822, APC, G 1-12: 90.

3 [ J. Monk ], "Notes sur un projet de bill à l'effet d'unir les législatures provinciales du Bas et du Haut-Canada" (31 mai 1822), APC, BasCanada (Q), 163-1: 177-178; aussi dans: Report on Canadian Archives 1897 (Ottawa, 1898), 11-12; [Anonyme, J. Monk], "Tenures, notes au sujet du $29 \mathrm{e}$ article" [s.d. juin 1822], APC, Q 163-1: 126; Monk à Wilmot, 2 juillet 1822, APC, Q 163-1: 94; J. Monk à R. Wilmot, 30 juillet 1822, $A P C, Q$ 162-2: 289; aussi à: G 1-12: 91-94.

4 Acte du Commerce du Canada, 5 août 1822, DRHC, 1819-1828, 121.

$5 \mathrm{~W}$. Horton à Ellice, 6 août 1823, APC, Q 175: 57-58. 
Ellice revient donc à la charge ${ }^{6}$ et la loi de 1822 est complétée en 1825, par l'Acte des tenures du Canada, qui permet aux seigneurs de racheter les droits que la Couronne a sur eux et de devenir propriétaires absolus de leurs terres non encore concédées (avec les précisions nécessaires cette fois), mais qui ne permet aux censitaires des seigneuries autres que celles de la couronne de se libérer que si leur seigneur a lui-même d'abord opéré la commutation avec la Couronne. ${ }^{7} \AA$ peine deux ou trois seigneurs se prévaudront de cette loi.

A cette époque, il y a des foyers de mécontentement dans les seigneuries dont les propriétaires sont exigeants, mais la masse du peuple demeure favorable à la tenure seigneuriale. Vers 1830 , le vent commence à tourner et, en 1837, c'est une vague qui déferle. ${ }^{8}$ Il n'y a pas nécessairement de raison tangible à cela, sauf dans les seigneuries où les seigneurs sont exigeants. Dans certains cas, on demande l'abolition des lods et ventes et des cens et rentes comme on demande l'abolition des dîmes; c'est le vent révolutionnaire qui souffle. Moses Hart le constate en ces termes: "Une grande clameur s'est élevée contre les seigneurs, quoiqu'en général, ils aient traité leurs censitaires d'une manière généreuse." ${ }^{9}$ A-t-il forcé la note en disant "en général"? C'est possible. Mais il reste une part de vérité non négligeable dans cette assertion. À St-Eustache, les chefs révolutionnaires font marcher leurs hommes en leur promettant d'abolir les redevances seigneuriales. ${ }^{10}$ Robert Nelson fait la même promesse au peuple en 1838. Et quand ces chefs promettent d'abolir les lods et ventes et les cens et rentes, ils veulent réellement dire ôter sans donner de compensation, décla-

${ }^{6}$ E. Ellice à R. W. Horton, 1 octobre 1824, APC, Q 175: 53.

76 Geo. IV, Ch. 59; "Acte des Tenures du Canada", 22 juin 1825, APC, Q 175-1: 2.

8 Agricola, "Correspondance", La Minerve, 10-94 (5 janvier 1837), $2 \mathrm{e}$ p., $3 \mathrm{e}$ col.

9 No 1, "Lettre de Moses Hart", 4 juin 1842, Rapport des Commissaires nommés pour s'enquérir de l'état des lois et autres circonstances qui se rattachent à la tenure seigneuriale dans le Bas-Canada, et appendice, 142.

10 Montreal Herald, 32-12 (28 janv. 1840), 2e p., 5e col.; tiré du Journal Historique des evenemens arrives a St. Eustache, par un témoin oculaire. 
rer que cela n'existe plus. La proclamation de Robert Nelson dit que la tenure seigneuriale est abolie comme si elle n'avait jamais existé dans ce pays et que tous ceux qui aideront la cause de la rébellion seront déchargés de leurs arrérages envers leurs seigneurs. ${ }^{11}$ Ces promesses d'abolition sans indemnité aux seigneurs flattent le peuple. Ce dernier en vient à rêver d'une vie plus facile où il n'aurait plus rien à payer au seigneur. Il se fait à l'idée que les charges seigneuriales peuvent être supprimées d'un trait de plume de la part du gouvernement et qu'il est possible d'obtenir ce résultat à force de le demander.

La nomination de Durham est un rayon d'espoir pour les Canadiens d'expression anglaise qui n'ont pas l'avantage d'être seigneurs. Dès avant son départ d'Angleterre, Durham est pris d'assaut par le Montréal britannique, qui réclame à cor et à cri l'abolition de la tenure seigneuriale dans le Bas-Canada, surtout dans Montréal. ${ }^{12}$ Une bonne partie d'entre eux consentent à verser une compensation, mais un groupe d'extrémistes entendent ne rien donner en échange. ${ }^{13}$ Les conseillers de Durham, T. E. M. Turton et Charles Buller, sont chargés d'étudier la question. Se basant sur le rapport des commissaires royaux de $1835,{ }^{14}$ ils rédigent un plan de commutation pour Montréal, qui reçoit l'approbation du Séminaire et des notables du groupe britannique. ${ }^{15}$ Ce plan est voté par le Conseil spécial sous forme d'Ordonnance à la demande de Thomson, en $1840 .^{16}$ Cette loi

11 M. Brunet, G. Frégault, M. Trudel, éd., Histoire du Canada par les textes, 159 .

12 N. C. Radiger à Durham, 24 mars 1838, APC, Durham 6, 1-1: 351; Pétition de l'Association constitutionnelle de Montréal, 4 avril 1838, APC, Q 255-2: 414, 417, 418; G. Moffatt et W. Badgley à Glenelg, 5 avril 1838, APC, Q 255-2: 396; Adresse des habitants d'origine britannique du BasCanada à la Reine, janvier 1838, APC, Q 255-2: 401-408; aussi dans: Rapport sur les Archives publiques pour l'année 1931, 500-502.

13 "Pétition des habitants britanniques et autres résidant dans la ville et les environs de Montréal", 21 juillet 1838, APC, Durham 5-1: 605. 14 "Rapports des Commissaires du Canada. Cinquième Rapport", 24 octobre 1836. Le Canadien, 6-151 (1 mai 1837), 1ère p., 1ère col.

$15 \mathrm{G}$. Buller, "Report from the Chief Secretary, on the Commutation of the Feudal Tenures in the Island of Montreal, and other Seigniories in the Possession of the Seigniory of St. Sulpice of Montreal", 31 octobre 1838, APC, Q 263: 391; i.e. Report on the Affairs of British North America, from the Earl of Durham, Her Majesty's High Commissioner, etc., février 1839, Appendice E, 185.

163 Vict. Ordonnances 1840 , ch. 30. 
autorise tous les tenanciers des trois seigneuries du Séminaire de Montréal qui le désirent, à racheter tous les droits seigneuriaux à des conditions libérales. 1840 restera une date extrêmement importante pour Montréal, parce que c'est cette année qui vit la libération du grand commerce et de l'industrie des entraves des lods et ventes. Et cette loi de 1840 est due aux demandes des industriels et des marchands d'origine britannique de Montréal.

Dès la première session du premier parlement du CanadaUni, en 1841, le député de Beauharnois, dont les électeurs avaient beaucoup à souffrir de leur seigneur, demande une enquête sur l'opportunité de changer les lois relatives à la tenure des terres et sur la façon la plus juste et la plus équitable d'effectuer les changements qui pourraient être jugés nécessaires. ${ }^{17}$ Il est exaucé. Les commissaires enquêteurs constatent que "la grande masse de la population Anglaise est en faveur d'une commutation, et paraît disposée à donner une indemnité raisonnable aux seigneurs", mais "que les habitans d'origine Française n'ont pas un très vif désir de changer la tenure de leurs terres si cette mesure tend à introduire des changemens dans les lois qui affectent leurs propriétés, quoiqu'ils désirent beaucoup se libérer des charges seigneuriales. Ils désirent se décharger des fardeaux qui pèsent sur eux avec le plus d'oppression, mais ils ne témoignent que rarement la volonté de donner un équivalent." Par ailleurs, les commissaires croient "qu'il est impossible, sans pousser loin l'exercice du pouvoir, de modifier la Tenure Seigneuriale, de manière à rencontrer les vues de la population franco-canadienne".

La conclusion générale de leur rapport est la suivante:

$1^{\circ}$ La majorité des censitaires, même s'ils ne demandent pas à racheter les droits seigneuriaux, se plaignent soit des charges seigneuriales, soit des abus des seigneurs.

$2^{\circ}$ Une moitié veut un changement de tenure, l'autre, une modification de la tenure existante.

${ }^{17}$ Journaux de l'Assemblée législative de la Province du Canada, 1: 103-104, 273. 
$3^{\circ}$ Essayer de faire marche arrière, c'est-à-dire de corriger les abus et d'amender la tenure seigneuriale, est impossible, à cause des jugements des tribunaux.

$4^{\circ}$ Il ne reste qu'une solution: faire disparaître les droits seigneuriaux.

$5^{\circ}$ Mais un grand nombre de censitaires veulent être déchargés sans qu'il leur en coûte ou si peu que rien. D'ailleurs, la plupart n'ont pas les moyens de racheter les droits seigneuriaux.

$6^{\circ}$ D'autre part, la justice exige que les seigneurs soient indemnisés pour la perte de leurs droits.

$7^{\circ} \mathrm{Si}$ les seigneurs ont violé la loi, en imposant certaines charges, ils n'ont pas droit à une indemnité, mais s'ils ont acheté leur seigneurie à haut prix, en s'appuyant sur les jugements des tribunaux, il serait injuste de les dépouiller sans leur donner de compensation.

$8^{\circ}$ On ne devrait pas donner aux seigneurs la propriété absolue de leurs terres non concédées.

$9^{\circ}$ Une commutation facultative n'aura presque pas d'effet, ceux qui ont le plus à se plaindre actuellement étant également ceux qui auraient la plus forte indemnité à verser pour racheter les droits seigneuriaux.

$10^{\circ}$ Enfin, une commutation aux frais de la Province mettrait cette dernière en faillite. ${ }^{18}$

Ce rapport est connu de la Chambre en 1843, mais il n'a pas de suite.

Au cours de la session de 1844-45, Robert Christie tente d'obtenir de la Chambre une loi de commutation obligatoire, ${ }^{19}$

18 A. Buchanan, J.-A. Taschereau, James Smith, "Rapport des Commissaires nommés pour s'enquérir de l'état des lois et autres circonstances qui se rattachent à la tenure seigneuriale dans le Bas-Canada, et appendice, 12.

19 Journaux de l'Assemblée législative de la province du Canada, 4 (1844-45), 63. 
mais on est tellement convaincu que ce n'est pas cela que le peuple veut, et que l'on sait si bien que forcer les censitaires à racheter les droits des seigneurs équivaudrait à en mettre les 4/5 dans le chemin, qu'on transforme le projet de loi de façon à permettre à ceux qui le désirent de racheter les droits seigneuriaux, sans y obliger personne. ${ }^{20}$ Le censitaire qui désire changer la tenure de sa terre, devra s'entendre avec son seigneur sur le montant qu'il aura à verser pour se libérer. ${ }^{21}$ Voilà une nouvelle loi visant à l'abolition du régime seigneurial. Pourtant, personne ne se prévaut de cette loi.

Robert Christie revient à la charge en 1847 , avec un projet de loi destiné à obliger les seigneurs à consentir au rachat de leurs droits par les censitaires lorsque ces derniers le demanderont, mais le projet ne reçoit pas l'appui de la Chambre. ${ }^{22}$

Sur les entrefaites naît, apparemment au sein de l'Institut canadien, nouvellement fondé, l'“Association pour la réforme des droits seigneuriaux", (qu'on remarque bien le mot réforme) laquelle entreprend une campagne systématique d'action sur l'opinion publique, en vue d'amener le peuple à réclamer une action de la part des gouvernants. ${ }^{23}$ Les députés Jean-Baptiste Mongenais, Pierre Davignon et Thomas Boutillier deviennent les principaux porte-parole de l'Association et distribuent à gauche et à droite les discours à l'emporte-pièce. Le peuple répond à leur appel. 1848 et 1849 sont des années de pétitions à la législature. 32,700 personnes demandent soit une réforme soit l'abolition de la tenure seigneuriale. ${ }^{24}$ A la surprise générale, LaFontaine refuse d'agir.

Voici comment se pose le problème. "L'Association pour la réforme des droits seigneuriaux", ainsi que la majorité des Canadiens français de la Chambre d'Assemblée, veulent, en se

20 Ibid., 278.

21 Ibid., 438, 443, 446.

22 Journaux de l'Assemblée législative de la province du Canada, 6 (1847), (Montréal, 1847), 10, 181.

23 "Association pour la réforme des droits seigneuriaux", La Minerve, 21-6 (28 sept. 1848), 2e p., 4e col.

24 Pierre Davignon, "Aux Signataires de la Requête des Comtés de Rouville, Chambly et Laprairie demandant la réforme de la tenure seigneuriale", La Minerve, 21-95 (6 août 1849), 2 e p., $6 \mathrm{e}$ col. 
basant sur la Coutume de Paris, les Arrêts de Marly et les jugements des intendants, faire déclarer illégaux les taux de cens et rentes supérieurs à deux sous par arpent et illégales nombre de charges imposées après la Conquête, d'abord par les seigneurs anglais, ensuite par quelques seigneurs canadiens. Une telle loi serait extrêmement dure, la ruine peut-être, pour les seigneurs de langue anglaise et pour certains seigneurs canadiens-français haut placés (conseillers législatifs ou exécutifs) qui les ont imités. Mais les censitaires ne veulent pas payer pour se débarrasser de charges que les seigneurs n'avaient même pas le droit de leur imposer. Et ils veulent savoir ce qui est légal. Est-ce la Coutume de Paris et les Arrêts de Marly, ou les décisions des cours de justice où siégeaient des juges seigneurs? De 1848 à 1854, les censitaires vont remuer ciel et terre pour obtenir une loi déclaratoire - rétroactive par conséquent - contre les seigneurs qui se sont montrés exigeants (et qui sont surtout des Anglais).

À l'automne de 1849, le docteur Pierre Davignon invite tous les censitaires de la Province à envoyer des représentants à Montréal, pour s'entendre sur un plan de projet de loi à soumettre à la législature. ${ }^{25}$ Les districts de Montréal et de TroisRivières répondent à l'appel. (Le district de Québec ne répond pas parce que le besoin de réforme s'y fait beaucoup moins sentir.) Les 120 délégués demandent en premier lieu une loi déclaratoire pour fixer les droits des seigneurs et ensuite l'abolition de la tenure seigneuriale. ${ }^{26}$ Le député Pierre Davignon, nommé président de la "Convention anti-seigneuriale de Montréal" (remarquez qu'on change le nom de l'association), est chargé d'arracher ces lois au gouvernement.

Pendant la session de 1850, LaFontaine, qui est au courant de ces faits et qui ne veut pas de loi déclaratoire, présente luimême deux résolutions sur la tenure seigneuriale. En les soumettant à la Chambre, il dit: "il y a actuellement des gens qui $2 \mathrm{e} \mathrm{col}$.

25 "Tenure seigneuriale", La Minerve, 22-1 (10 sept. 1849), 3e p., 5e col.

26 "Tenure seigneuriale", La Minerve, 22-10 (11 nov. 1849), 2e p., 
pensent que la tenure seigneuriale doit être abolie purement et simplement sans indemniser les seigneurs... Mais plus cette opinion est forte, plus la chambre doit se prononcer avec fermeté pour montrer aux parties intéressées qu'il ne peut y avoir de commutation sans indemnité au seigneur pour toute la valeur de ses droits. (...) Cela ne peut être effectué sans que la commutation soit obligatoire". ${ }^{27}$ La Chambre adopte les résolutions de LaFontaine, dont la première dit: "il importe... d'effectuer, à une époque aussi rapprochée que possible, la conversion de "la tenure seigneuriale" en une tenure libre, en protégeant et réglant équitablement tous les intérêts concernés." La seconde se lit comme suit: "la dite commutation ne peut avoir lieu qu'au moyen d'une indemnité suffisante en faveur de tous ceux dont les justes droits seront lésés en l'effectuant." 28

Pierre Davignon présente sa motion de loi déclaratoire, visant à "établir la ... dite tenure dans sa pureté primitive, conformément aux anciennes lois ... du Bas-Canada". ${ }^{29}$ Cartier supplie Davignon de se contenter des résolutions de LaFontaine, pour trois raisons: $1^{\circ}$ parce que l'adoption d'une loi déclaratoire ne serait pas chose facile; $2^{\circ}$ parce qu'une telle loi ne ferait pas disparaître la tenure seigneuriale; $3^{\circ}$ parce que la résolution Davignon ne porte que sur les cens et rentes, alors que ce sont les lods et ventes qui constituent le principal inconvénient de la tenure seigneuriale. ${ }^{30}$ Il se passe un échange de mots aigredoux entre Davignon et LaFontaine. Finalement, voyant que LaFontaine est intraitable, et pour éviter de renverser le ministère, Davignon modifie sa résolution de façon à ce qu'elle ne soit plus qu'une demande d'étude de la question par un comité. ${ }^{31}$

27 "Chambre d'Assemblée", 14 juin 1850, La Minerve, 22-83 (20 juin 1850), 2e p., 1ère col.

28 Journaux de l'Assemblée législative de la province du Canada, 9 (1850), 96.

29 "Tenure Seigneuriale", La Minerve, 22-82 (17 juin 1850), 1ère p., 5e col.

30 "Tenure seigneuriale", 25 juin 1850, La Minerve, 22-86 (1er juill. $1850), 2 \mathrm{e}$ p., $5 \mathrm{e}$ col.

31 "Chambre d'Assemblée", 26 juin 1850, La Minerve, 22-87 (4 juillet $1850), 2 \mathrm{e}$., 3e col.; Journaux de l'Assemblée légisiative de la province du Canada, 9 (1850), 97. 
Le comité chargé de cette étude ne peut arriver à une conclusion avant la fin de la session. Il reprend son travail avec la session de $1851,{ }^{32}$ et, cette fois, recommande l'adoption d'une loi déclaratoire fixant les droits des seigneurs, et présente un projet de loi à cette fin. ${ }^{33}$ LaFontaine, très mécontent, s'emporte et dit qu'on n'a pas demandé un tel projet de loi au comité, mais bien un plan d'abolition de la tenure seigneuriale. ${ }^{34}$ L'honorable Jean Chabot avertit LaFontaine que la Chambre n'approuvera pas ses allégations contre le comité. Tentant de régler le conflit, Baldwin suggère que le comité prépare un projet d'abolition et que la Chambre l'adopte de pair avec le premier. ${ }^{35}$ Le comité se réunit et, à son corps défendant, présente un projet de commutation visant à faire disparaître la tenure seigneuriale dans les cinq ans à venir. ${ }^{36}$ Toutefois, il fait remarquer que le seul projet de loi qui devrait être adopté pour le moment, est celui de la loi déclaratoire. ${ }^{37}$ Comme LaFontaine s'oppose à une telle loi, après la seconde lecture des deux projets de lois, il clot la session, fait dissoudre le Parlement et se retire de la politique.

La tenure seigneuriale fait le thème de la campagne électorale qui suit. Les candidats des deux partis s'engagent à régler la question de la tenure seigneuriale. Quatre membres de la "Convention anti-seigneuriale de Montréal" sont élus députés. ${ }^{38}$

$\mathrm{Au}$ début de la session de 1852-53, une nouvelle vague de pétitions contre la tenure seigneuriale déferle sur la législature.

32 Journaux de l'Assemblée législative de la province du Canada, 10 (1851), (Québec, 1851), 21.

33 Appendice du dixième volume des journaux de l'Assemblée législative de la province du Canada, 4 (1851), Appendice NNN, Appendice A, [n.p.], 26e p., 2e col.; Aussi dans La Minerve, 23-108 (16 août 1851), lère $\mathrm{p} ., 5 \mathrm{e}$ col. $4 \mathrm{e}$ col.

34 "Tenure seigneuriale", La Minerve, 23-113 (28 août 1851), 2e p. $4 \mathrm{e}$ col. 5 e col

35 "Assemblée législative", La Minerve, 24-114 (30 août 1851), 2e p., 36 "Droits seigneuriaux", La Minerve, 23-116 (4 sept. 1851), 1ère p.,

37 Journaux de l'Assemblée législative de la province du Canada, 10 (1851), 325.

38 D. Latte, "Convention anti-seigneuriale de Montréal", La Minerve, 26-148 (31 août 1854), 2e p., 6e col. 
Le solliciteur général Lewis Thomas Drummond présente un projet de loi dont la première partie ressemble au projet de loi déclaratoire présenté l'année précédente, et dont la seconde indique de quelle façon s'opérera le rachat des droits seigneuriaux pour ceux qui le désireront. ${ }^{39}$ Mais le projet de loi de 1852 est plus astucieux que celui de l'année précédente. Il ne s'agit plus d'une loi déclaratoire, en ce sens qu'on ne prétend pas dire ce que la loi a été par le passé et par conséquent ce qu'elle est encore à ce jour, mais on décide, en tant que législateur, ce qu'elle sera à l'avenir. Ainsi, le maximum des rentes est fixé désormais à 4 sous par arpent. Les tribunaux décideront si les rentes supérieures à cette somme étaient auparavant légales ou non. Si oui, le gouvernement indemnisera les seigneurs pour la perte qu'ils subiront par suite de la diminution des rentes. On voit que la pensée du gouvernement s'est modifiée dans le sens de la préservation des droits des seigneurs. Ces derniers viennent de gagner une première manche: ils ont fait accepter le principe du recours aux tribunaux et un grand nombre de juges sont des seigneurs.

La deuxième partie du projet de loi est également bien différente du projet de commutation présenté en 1851 à la demande de LaFontaine. Celui de 1851 visait à l'abolition de la tenure seigneuriale dans les 5 ans, alors que celui de 1852 permet à cette tenure (astreinte aux conditions spécifiées dans la première partie du projet de loi), de continuer indéfiniment. Cependant, tout censitaire peut se libérer quand il le voudra et toute seigneurie peut se libérer en bloc, à condition que les $2 / 3$ des censitaires de cette seigneurie en fassent la demande.

Pendant l'étude en comité, le taux maximum des rentes est porté à $3 \frac{1}{2}$ sous. ${ }^{40}$ Le Conseil législatif ne délibère pas plus d'une demi-heure sur le projet de loi avant de le rejeter. ${ }^{41}$

39 Journaux de l'Assemblée législative de la province du Canada, 11-1 (1852-53), 233.

40 "Tenure seigneuriale. Amendement au bill seigneurial", La Minerve, 25-78 (16 avril 1853), $2 \mathrm{e}$ p., $4 \mathrm{e}$ col.

41 Journaux du conseil législatif de la province du Canada, 11 (1852), (Québec, [1853]), 527; "Tenure seigneuriale", La Minerve, 25-98 (2 juin 1853), 2e p., 6e col. 
Entre la session de 1852-53 et celle du début de l'été 1854, deux des membres les plus actifs de la "Convention anti-seigneuriale de Montréal", le français D. Latte et Joseph Doutre, organisent des assemblées anti-seigneuriales dans tous les coins de la Province, et font adopter par les gens des résolutions contre la tenure seigneuriale, qu'ils publient ensuite dans les journaux. Leur but est de tenir l'opinion publique en alerte et les hommes politiques en haleine. Ils y réussissent parfaitement. ${ }^{42}$

Le ministère Hincks-Morin, auquel lord Elgin demande de ne pas toucher à la question seigneuriale au cours de la session qui vient, retarde le début de celle-ci jusqu'au 13 juin et annonce qu'elle sera courte et qu'on n'y étudiera pas la question des tenures. ${ }^{43}$ Résultat: dès les premiers jours, le ministère est renversé, la session est prorogée et le parlement dissous. ${ }^{44}$

Dans le Bas-Canada, l'élection de 1854 se fait exclusivement sur la question de la tenure seigneuriale. Morin, qui a refusé de régler cette question, est défait dans sa propre circonscription, ${ }^{45}$ mais son parti, qui veut avant tout réformer la tenure seigneuriale et qui a, par deux fois déjà, présenté un projet de loi visant à mater les seigneurs, est reporté au pouvoir de préférence à l'opposition qui réclame l'abolition totale et immédiate de la tenure seigneuriale. Le résultat de cette élection prouve qu'en 1854, la majorité des gens voulaient non pas l'abolition de la tenure seigneuriale, mais une loi ramenant les droits des seigneurs à ce qu'ils avaient été sous le régime français.

Les Chambres sont convoquées pour le début de septembre (1854). Le discours du trône invite les législateurs à régler la

42 "Nouvelle convention pour l'abolition de la tenure seigneuriale", La Minerve, 25-116 (14 juillet 1853), $2 \mathrm{e}$ p., $4 \mathrm{e}$ col. $2 \mathrm{e}$ col.

43 "Chambre d'Assemblée", La Minerve, 26-117 (20 juin 1854), 2e p.,

44 "Nouvelles télégraphiques", La Minerve, 26-118 (22 juin 1854), 2e p., 5e col.; [Sans titre], La Minerve, 26-120 (27 juin 1854), 2e p., 6e col. 3e col.

45 "Comté de Terrebonne", La Minerve, 26-135 (1er août 1854), 2e p., 
question de la tenure seigneuriale en respectant le droit de propriété. ${ }^{46}$ Les ministres bas-canadiens demeurent les mêmes Morin s'étant fait élire dans une autre circonscription - et s'unissent à MacNab à la condition expresse que ce dernier règle la question de la tenure dans le sens prôné par leur parti, c'est-à-dire en faisant des réformes à la tenure seigneuriale. ${ }^{47}$

Le 20 octobre, le procureur général introduit un projet de loi dont le titre est: Bill "pour définir et limiter les droits seigneuriaux, pour en faciliter le rachat, et pour abolir les Lods et Ventes". ${ }^{48}$ Le parti ne démord pas de sa ligne de conduite: en premier lieu, mettre un frein aux exigences des seigneurs, en second lieu, autoriser les censitaires qui le désireront à racheter les droits seigneuriaux. Le procureur général Drummond, en présentant son projet de loi, dit expressément: “je pense que ce ne serait pas être l'ami du peuple que de l'obliger à commuer quand il ne le désirera pas. (...) L'administration cependant, ne fait pas de ce point une question ministérielle; quelques-uns de ses membres aimeraient mieux fixer un temps durant lequel le censitaire serait obligé de racheter les droits du seigneur. Pour moi, je désire ne pas limiter le temps. Ce principe est bien plus populaire que celui qui obligerait le censitaire à commuer qu'il le veuille ou non." Drummond confirme ici ce qu'avait révélé le résultat de l'élection de 1854, à savoir que la majorité des gens veulent seulement qu'on limite les droits des seigneurs et qu'on autorise simplement le rachat des droits seigneuriaux pour ceux qui le désirent. ${ }^{49}$

Le projet de loi adopté par l'Assemblée était conforme au vœu du peuple. 1ère col.

46 “Ouverture du parlement”, La Minerve, 27-1 (9 sept. 1854), 2e p.,

47 "Résignation du ministère. Discours de M. Hincks", La Minerve, 27-1 (9 sept. 1854), 2e p., 3e col.; "Parlement provincial", La Minerve, 27-2 (12 sept. 1854), 2e p., 1ère col.

48 Pétitions de P. Blanchet et autres, L. Guérin et autres, F. Nye et autres, S. Bertrand et autres, J. Troie, Junior et autres, B.C.A. Gugy, dans: Journaux de l'Assemblée législative de la province du Canada, 13 (1854-55), (3 parties), (Québec, 1855), 1: 208.

49 "Tenure seigneuriale: Discours de M. Drummond", La Minerve, 27-25 (7 nov. 1854), 2e p., 6e col. 
$1^{\circ}$ Il limitait l'étendue de terre que le seigneur avait le droit de se réserver pour son domaine.

$2^{\circ}$ Il réunissait au domaine de la Couronne toutes les terres non concédées des seigneuries. d'eau.

$3^{\circ}$ Il abolissait les droits des seigneurs sur les cours

$4^{\circ}$ Il abolissait le droit de banalité.

$5^{\circ} \mathrm{Il}$ abolissait les privilèges et honneurs réservés aux seigneurs.

$6^{\circ}$ Il abolissait le droit de retrait conventionnel.

$7^{\circ} \mathrm{Il}$ limitait les cens et rentes à deux sous par arpent en superficie.

$8^{\circ} \mathrm{Il}$ autorisait à remplacer toute redevance payable en nature ainsi que les corvées par une somme d'argent.

$9^{\circ}$ Il limitait les cas de vente par décret.

$10^{\circ}$ Il défendait de réclamer plus de cinq ans d'arrérages.

$11^{\circ}$ Il déclarait nulle toute obligation autre que celle de tenir feu et lieu; celle de faire arpenter aux frais du censitaire; celle d'imposer des cens et rentes n'excédant pas 2 sous.

$12^{\circ}$ Il permettait au censitaire de racheter les droits reconnus dans le projet de loi, c'est-à-dire les cens et rentes et les lods et ventes.

$13^{\circ} \mathrm{Il}$ abolissait les droits de mutation sur la vente des propriétés concédées après l'adoption de cette loi.

$14^{\circ}$ Il décrétait que la première vente de toute propriété concédée après l'adoption de cette loi, transformerait automatiquement les droits seigneuriaux en une rente constituée rachetable au gré du censitaire. 
$15^{\circ}$ Il autorisait les $2 / 3$ des censitaires d'une seigneurie à obtenir la transformation de toutes les redevances seigneuriales de la seigneurie en rentes constituées rachetables au gré de chaque censitaire.

$16^{\circ}$ Il limitait les réclamations d'arrérages de lods et ventes à 5 ans.

$17^{\circ}$ Il déclarait que tout fonds dont les redevances seigneuriales avaient été commuées en vertu de lois antérieures à la présente, était censé avoir été et était tenu en franc-alleu roturier (soumis par conséquent aux lois françaises) plutôt qu'en franc et commun soccage (lequel impliquait les lois anglaises).

$18^{\circ}$ Il prévoyait la création d'un tribunal spécial destiné à déterminer les droits des seigneurs.

$19^{\circ} \mathrm{Il}$ autorisait les seigneurs à présenter un état détaillé des pertes qu'ils auraient à subir par suite de l'adoption de cette loi. Des commissaires jugeraient si le gouvernement devait leur accorder une indemnité.

$20^{\circ}$ Il appliquait le fonds d'indemnité voté par la Province au paiement des rentes supérieures à deux sous par arpent en superficie.

Le conseil législatif, qui ne compte pourtant que deux seigneurs, modifie le projet de loi du tout au tout. Le projet accepté par l'assemblée mettait un frein à l'ambition des seigneurs et autorisait les censitaires à se libérer, mais ne les y obligeait pas: c'était un projet de commutation facultative. Le conseil en fait un projet de commutation obligatoire. Il substitue une rente constituée à toutes les charges seigneuriales. Il supprime à peu près toutes les restrictions que l'Assemblée avait voulu imposer aux seigneurs, laissant à ces derniers toutes leurs terres non concédées. Il applique le fonds d'indemnité au rachat des charges dans l'ordre suivant: $1^{\circ}$ des lods et ventes, $2^{\circ} \mathrm{du}$ droit de banalité, $3^{\circ}$ des cens et rentes excédant un sou et demi par arpent en superficie. ${ }^{50}$ 
Le gouvernement était acculé au pied du mur et ne pouvait plus reculer sans être renversé comme l'avait été le précédent, ni même retarder l'adoption de la mesure sans risquer de perdre l'appui du Haut-Canada relativement à l'indemnité fournie par le trésor public pour aider au rachat des droits des seigneurs. En effet, le Haut-Canada avait consenti à regret à donner son appui au Bas-Canada sur ce sujet, à la condition d'obtenir la collaboration du Bas-Canada pour son bill relatif aux réserves du clergé. Or ce dernier était adopté quand, avant l'ajournement de Noël, le projet de loi du Bas-Canada envoyé au conseil législatif comme un bill de réforme du régime seigneurial et de commutation facultative, en revint sous forme d'un projet d'abolition immédiate et de commutation obligatoire. Ne pouvant absolument pas faire autrement, l'Assemblée législative se désista de ses privilèges et accepta les amendements, autrement dit le nouveau projet de loi qui lui était présenté par le conseil législatif. ${ }^{51}$

Elgin s'empressa de donner l'assentiment royal à cet acte de législation, qui apportait une solution partielle à l'un des plus formidables casse-tête qu'ait jamais eus une législature. ${ }^{52}$

C'est ainsi qu'on eut une loi d'abolition alors que la majorité des censitaires auraient préféré, pour le moment du moins, une réforme de la tenure seigneuriale.

En résumé, les exigences très grandes de certains seigneurs ont soulevé l'opinion publique à un point tel que les élections se faisaient presque exclusivement sur la question de la tenure seigneuriale, que l'homme d'État qui refusait d'agir dans le sens voulu par le peuple y brisait sa carrière politique (ex. L.-H. LaFontaine) et que le ministère qui voulait contenir la marée sautait immédiatement (ex. le ministère Hincks-Morin au printemps de 1854).

Donc, lorsqu'on parle de l'abolition du régime seigneurial, il faut se rappeler que ce dernier n'a pas été aboli en même

\footnotetext{
51 Journaux de l'Assemblée législative de la province du Canada, 13 (1854-55), 1: 544 .

52 [Sans titre], La Minerve, 27-42 (19 déc. 1854), 2e p., 1ère col.
} 
temps partout, ni par une seule loi, et que, par conséquent, il faut spécifier de quel endroit on parle et de quelle loi il s'agit. Si c'est de 1854 qu'on parle, il faut dire que la loi appelée "L'Acte seigneurial de 1854" a été adoptée par suite des plaintes et des pétitions du peuple, lesquelles furent provoquées principalement par les exigences considérées à l'époque comme abusives de certains seigneurs, surtout de langue anglaise et de la région de Montréal. Il y a une phrase qui se retrouve dans la plupart des discours prononcés par les membres des deux Chambres au cours des débats sur ce sujet, c'est celle-ci: "Il faut faire quelque chose parce qu'il y a trop de plaintes, trop d'agitation dans la Province relativement à la question de la tenure seigneuriale."

C'est une vérité qu'un grand nombre de seigneurs ont traité leurs censitaires avec bonté. Mais c'est une autre vérité que s'il y a eu une loi d'adoptée en 1854 relativement aux droits seigneuriaux, c'est par suite des plaintes du peuple contre les exigences de certains seigneurs et parce que ces exigences avaient soulevé une clameur telle que nul homme d'Etat ni aucun gouvernement n'y pouvaient résister.

Collège militaire royal,

GeORges BAILlargeoN

Saint-Jean, Qué. 